\title{
Multi-optimization Criteria-based Robot Behavioral Adaptability and Motion Planning
}

\section{DOE Project Number: 82794}

\section{Research Objectives:}

Robotic tasks are typically defined in Task Space (e.g., the 3-D World), whereas robots are controlled in Joint Space (motors). The transformation from Task Space to Joint Space must consider the task objectives (e.g., high precision, strength optimization, torque optimization), the task constraints (e.g., obstacles, joint limits, non-holonomic constraints, contact or tool task constraints), and the robot kinematics configuration (e.g., tools, type of joints, mobile platform, manipulator, modular additions, locked joints). Commercially available robots are optimized for a specific set of tasks, objectives and constraints and, therefore, their control codes are extremely specific to a particular set of conditions. Thus, there exist a multiplicity of codes, each handling a particular set of conditions, but none suitable for use on robots with widely varying tasks, objectives, constraints, or environments. On the other hand, most DOE missions and tasks are typically "batches of one". Attempting to use commercial codes for such work requires significant personnel and schedule costs for re-programming or adding code to the robots whenever a change in task objective, robot configuration, number and type of constraint, etc. occurs.

The objective of our project is to develop a "generic code" to implement this Task-space to JointSpace transformation that would allow robot behavior adaptation, in real time (at loop rate), to changes in task objectives, number and type of constraints, modes of controls, kinematics configuration (e.g., new tools, added module). Our specific goal is to develop a single code for the general solution of under-specified systems of algebraic equations that is suitable for solving the inverse kinematics of robots, is useable for all types of robots (mobile robots, manipulators, mobile manipulators, etc.) with no limitation on the number of joints and the number of controlled Task-Space variables, can adapt to real time changes in number and type of constraints and in task objectives, and can adapt to changes in kinematics configurations (change of module, change of tool, joint failure adaptation, etc.).

\section{Research Progress and Implications:}

Our overall approach is based on a novel method for the resolution of under-specified systems of algebraic equations, involving both a novel formulation of the general solution as:

and a Lagrangian-based constrained optimization

$$
\begin{gathered}
S=\left\{\Delta \bar{q} \in \mathfrak{R}^{m}, \Delta \bar{q}\left(t_{1}, \ldots, t_{m-n+1}\right)=\sum_{i=1}^{m-n+1} t_{i} \bar{g}_{i}, \sum_{k=1}^{m-n+1} t_{k}=1\right\} \\
L\left(t_{i}, \mu, v_{j}\right)=Q\left(t_{i}\right)+\mu\left(\sum_{i=1}^{m-n+1} t_{i}-1\right)+\sum_{j=1}^{r} v_{j} C^{j}\left(t_{i}\right)
\end{gathered}
$$

where

$$
Q\left(\Delta \bar{q}\left(t_{i}\right)\right), i=1, m-n+1
$$

is the general criterion to be optimized, and

$$
C^{j}\left(\bar{q}, \Delta \bar{q}\left(t_{i}\right)\right)=0, j=1, r ;
$$


represents a set of $r$ general constraints. The unique aspect of this approach is that it leads to analytical solutions that simultaneously fulfill the optimization criterion and all constraints applicable at the particular time-step (loop rate) $[1,2,3]$.

As of the first nine months of this 3-year project, we have completed a preliminary version of the code for the solution space and optimization based on least norm criteria. This code was verified for proper resolution in comparisons with the standard Moore-Penrose pseudo-inverse in several sample problems involving manipulators and mobile manipulators with $6,7,10$, and 13 d.o.f. The code was also verified for proper handling of constraints that could change at loop-rate, including avoidance of multiple obstacles and joint limits. The significance of these successful accomplishments is that they indicate the feasibility of the single-code concept to handle widely varying type and numbers of constraints and criteria, that can change at loop rate, through the novel optimization scheme.

We also have completed the algorithm and a test version of a code for automatically generating the forward kinematics equations of modules and complete manipulator systems from the set of Denavit-Hartenburh (DH) parameters representing each module. The algorithm proceeds through automatic generation of the transformation matrices representing the modules, automatic generation of the global transformation matrix of the complete system, and extraction of the forward kinematics equations, in symbolic form. From this symbolic representation, automated differentiation is performed to automatically generate, in symbolic form, the Jacobian matrix for the overall system. This algorithm and code have been tested for proper processing on a variety of modules and complete robotic systems. The implication of this successful automated generation of the forward kinematics equations and Jacobian matrix for any manipulator joint-link module is the feasibility of the "genericness" of the single-code, i.e., the capability for the single code to handle robots that can change configuration during tasks (e.g., when acquiring or changing tools) and robots that are modular.
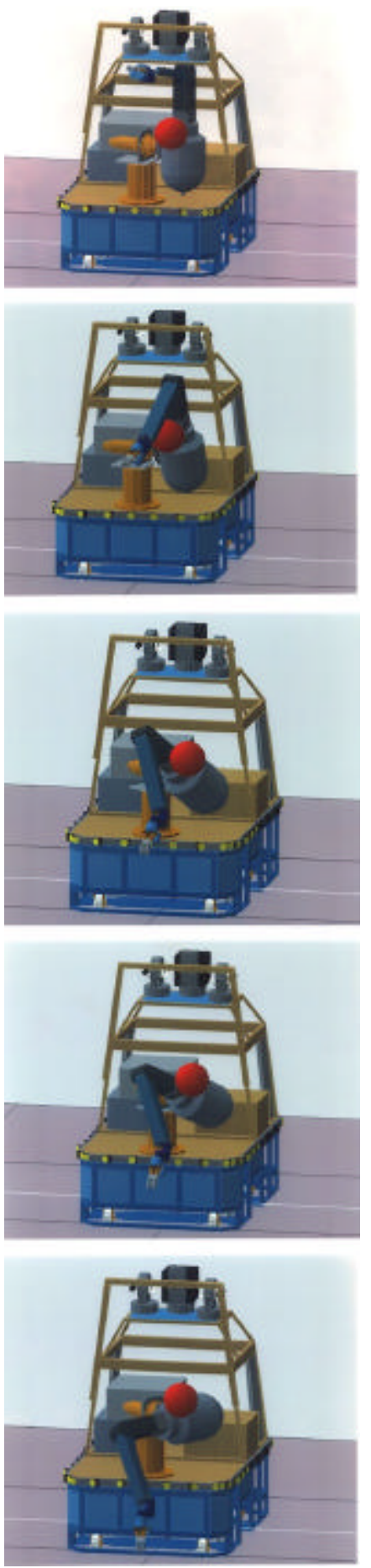

Constraints being handled at loop rate during a sample trajectory. 


\section{Planned activities:}

During the remainder of this first year of activities, the overall resolution framework and code architecture will be completed to allow the motion planning module to be suitable for use directly in both simulation mode and in actual target robot control hardware. The final code module for automated adaptation to kinematics configuration changes will also be completed. Analytical solutions approaches for handling, in bounded algorithmic time, novel sets of constraints, in particular those related to tool operation (e.g., linear motion for a drill, planar motion of a saw, etc.) will be derived for inclusion in the overall resolution code. In FY-03, our activities will focus on automated methods for direct feedback of changing task conditions (e.g., when changing objective function, changing tool, changing constraints, etc.) into the resolution code from either sensory or human input to achieve behavior adaptation through change of criteria. With completion of the integrated version of the complete code, we will then perform extensive computational experiments to establish characteristic orders, complexity, and completeness of the algorithms. In FY-04, we will focus on investigations of non-linear task objective criteria and corresponding analytical resolution. For each solution strategy and implementation, we will perform experimental investigations of complexity and bounded time through comparative computational experiments to assess preferred resolution approaches for these objective functions. We will also focus on proof-of-principle experiments using kinematics configurations of existing D\&D robots at ORNL, including implementation on an actual robot control system to demonstrate pervasiveness of the single-code approach.

\section{Information access:}

Further background and details of the methodologies can be found in the following publications:

1 - F. G. Pin et al., Journal of Robotic Systems 13 (11), 723-736 (1996))

2 - F. G. Pin and F. A. Tulloch. Proceedings of the 1996 IEEE International Conference on Robotics and Automation, Minneapolis, Minnesota, April 22-28, 1996, Vol. 1, 468-473

3 - F. G. Pin et al. Proceedings of the IEEE International Conference on Robotics and Automation, San Diego, California, May 8-13, 1994, 96-102.

4 - F. G. Pin et al. Proceedings of the 1997 IEEE International Conference on Robotics and Automation, Albuquerque, New Mexico, April 21-27, 1997, Vol. 4, 2914-2919. 